\title{
PROJETO DE MENTORIA ONLINE COMO AUXÍLIO AO DESENVOLVIMENTO DE CARREIRA NA ENGENHARIA
}

Ana L. B. Primo - aluizabotelho@ieee.org*

Universidade Federal do Pará, Faculdade de Eng. da Computação e Telecomunicações*

R. Augusto Corrêa, 01 - Guamá*

66075-110-Belém-Pará*

Shirley K. S. Ferreira - shirley.ferreira@itec.ufpa.br*

Universidade Federal do Pará, Programa de Pós-Graduação em Engenharia Elétrica*

R. Augusto Corrêa, 01 - Guamá*

66075-110-Belém-Pará*

Resumo: Uma importante questão abordada no ensino em engenharia é expandir a visão dos(as) discentes para além da sala de aula e desenvolver outras habilidades, além das técnicas. Dessa forma, visando contribuir com o desenvolvimento de carreira profissional de membros(as) IEEE, voluntários(as) e da comunidade acadêmica como um todo, foi desenvolvido o projeto Mentoria de Carreira Universitária pelo grupo de afinidade Mulheres na Engenharia IEEE UFPA. Totalmente online, este projeto abordou, por meio de lives no YouTube, assuntos relacionados às habilidades e competências importantes que uma empresa busca, os processos seletivos de estágio e projetos acadêmicos que contribuem para o desenvolvimento e crescimento profissional dos(as) discentes de engenharia. Como resultado, ao longo de um mês foram alcançadas 2400 visualizações, com onze lives divididas entre webinars, workshops e cafés tecnológicos, todas promovidas por engenheiras e alunas de engenharia.

Palavras-chave: IEEE. Carreira Profissional. Mentorias.

\section{INTRODUÇÃO}

O uso de ferramentas digitais no compartilhamento de conhecimento já é uma realidade. As Novas Tecnologias da Informação e Comunicação (NTICs) incorporam a interação e comunicação em tempo real e impactam diversos âmbitos da sociedade, em especial a educação, em que cada vez mais se observa o uso de plataformas de compartilhamento de materiais, videoaulas e a promoção de Educação a Distância (EAD) (RODRIGUES, 2020, SILVA, ALMEIDA, 2019). Além disso, com o cenário singular que se vive no ano de 2020 devido à pandemia do novo coronavírus, o uso dessas tecnologias aumentou como alternativa para diminuir os impactos causados pelo fechamento de escolas e universidades.

No que concerne ao aprendizado durante a graduação, é diferencial que o(a) aluno(a) seja capaz de expandir sua visão além da sala de aula e desenvolver habilidades essenciais para a carreira profissional, além das técnicas e teóricas. Dessa forma, obter orientações de quem já está atuante no mercado de trabalho é uma alternativa de simples promoção e que pode ser decisivo na carreira de um estudante. Neste contexto, a mentoria cria uma estrutura de suporte aos alunos que se encontram em início de carreira, em que, profissionais atuam como conselheiros aos mentorados, dando suporte no que concerne ao planejamento de estratégias de 
carreira e ao desenvolvimento de competências e habilidades essenciais dentro do mercado de trabalho (ARANTES, VIEGAS, 2018).

Aliada à mentoria, tem-se também a metodologia de tornar o aprendizado envolvido mais dinâmico e até lúdico. Por exemplo, utilizar elementos de jogos para difundir conhecimento e treinamento aos participantes de determinado processo educativo, como em uma mentoria, define a chamada gamificação (TOLOMEI, 2017). Este tipo de estratégia auxilia o engajamento e dedicação dos mentorados, como será tratado neste projeto.

Dentro deste contexto, o presente trabalho aborda o projeto intitulado Mentoria de Carreira Universitária, desenvolvido com o objetivo de não somente auxiliar os(as) alunos(as) de diversas áreas da engenharia, como também atuar como uma ferramenta adicional dentro do contexto da pandemia, já que as aulas e demais atividades foram suspensas na universidade. $\mathrm{O}$ projeto realizado durante todo o mês de julho de 2020, foi idealizado pelo grupo Mulheres na Engenharia (WIE do inglês Women in Engineering), da Universidade Federal do Pará (UFPA), consistindo em uma representação do Instituto dos Engenheiros Eletricistas e Eletrônicos (IEEE), que se faz presente na UFPA desde 2013.

O IEEE é uma organização técnica a nível mundial e sem fins lucrativos, cujo objetivo é a difusão de tecnologias e conhecimentos em benefício da humanidade (IEEE, 2020). Nas universidades, o IEEE pode ser representado por seus membros (alunos(as) e professores(as)) e pelos chamados Ramos Estudantis, Capítulos Técnicos e Grupos de Afinidades. Desde sua fundação na UFPA, o Ramo Estudantil IEEE UFPA tem promovido uma série de atividades técnicas e extensionistas, com o objetivo de aproximar os(as) discentes da carreira profissional.

\section{MATERIAIS E MÉTODOS}

\subsection{Temáticas abordadas}

O objetivo principal do projeto Mentoria Universitária foi promover workshops online e compartilhar trajetórias de engenheiras de sucesso, sejam já formadas ou ativas dentro da universidade por meio de projetos de pesquisa e extensão.

Os temas abordados no projeto estão descritos no Quadro 1.

\begin{tabular}{|c|c|}
\hline Quadro 1 - Temas trabalhados no projeto \\
\begin{tabular}{|c|c|}
\hline Tema & Número de Palestrantes \\
\hline Movimento Empresa Júnior & 1 \\
\hline Workshop: Estágio na Prática & 1 \\
\hline Café Tecnológico com Engenheira de Alimentos (I) & 1 \\
\hline Networking no IEEE & 1 \\
\hline Pesquisa Científica & 1 \\
\hline Workshop: Currículo e LinkedIn & 1 \\
\hline Café Tecnológico com Engenheira de Alimentos (II) & 3 \\
\hline Competições Universitárias & 1 \\
\hline
\end{tabular}
\end{tabular}




\begin{tabular}{|c|c|}
\hline Workshop: Trainee na Prática & 1 \\
\hline Café Tecnológico com Engenheira Biomédica (III) & 1 \\
\hline Extensão e Empreendedorismo Social & 1 \\
\hline Experiência Internacional & 1 \\
\hline Organização Financeira & 1 \\
\hline
\end{tabular}

Fonte: Autor (2020)

Estas atividades foram proporcionadas durante todo o mês de julho às segundas, quartas e quintas, seguindo um cronograma definido da seguinte forma: a primeira semana de projeto englobou as temáticas de Movimento Empresa Júnior, estágio na prática e café tecnológico I; a segunda semana englobou networking no IEEE, pesquisa científica, workshop de currículo e LinkedIn e o café tecnológico II; a terceira semana envolveu competições universitárias, workshop de trainee na prática e café tecnológico III, e, por fim, a quarta semana abordando sobre extensão e empreendedorismo social, experiência internacional e o workshop de organização financeira.

No fim de cada semana (às sextas-feiras) era enviado aos inscritos um questionário referente ao que foi abordado durante as lives, utilizando a plataforma Kahoot, com o objetivo de avaliar o que os estudantes puderam aprender durante os eventos. A plataforma Kahoot possibilitou desenvolver este questionário como uma espécie de game (perguntas e respostas), utilizando uma interface gráfica dinâmica com o usuário, em que cada pergunta valia pontos. Ao final do projeto, gerou-se um ranking geral e os 10 primeiros lugares, ou seja, os participantes que acumulavam mais pontos durante as semanas do projeto, foram premiados.

Esta metodologia de usar pontos para as perguntas e premiar os melhores colocados atuou como um elemento atrativo para os participantes e proporcionou o acompanhamento efetivo ao longo de todo o projeto, além de tornar a experiência divertida.

A seguir cada atividade é descrita.

I. Movimento Empresa Júnior: em forma de webinar, teve como objetivo discutir a promoção do empreendedorismo dentro das universidades por meio das Empresas Juniores. Foi abordado o que é uma EJ (Empresa Júnior) e o Movimento de Empresas Juniores (MEJ) no Brasil, os processos seletivos para fazer parte das empresas, como fundar uma EJ, a vida de um pós-júnior (integrante da EJ que já se formou) e o que contribui para um(a) estudante integrar uma EJ no período de graduação. Também foram explanadas quais habilidades são desenvolvidas e quais são os conhecimentos adquiridos em uma Empresa Júnior de Engenharia. As palestrantes eram integrantes de EJ da universidade.

II. Estágio na Prática: este workshop abordou como acontece todo o processo de estágio em engenharia, desde a preparação do(a) estudante, o desenvolvimento do currículo, a inscrição em sites de recrutamento, a aplicação às vagas, comportamento em entrevistas, até a seleção do(a) candidato(a) e sua carreira dentro da instituição que o(a) contratou, dando todo o panorama da experiência de ser estagiário(a). A palestrante era uma estudante de engenharia da computação e estagiária em uma grande empresa, já com experiências anteriores.

III. Café Tecnológico: o café tecnológico é um evento tradicional do ramo estudantil IEEE UFPA e seu principal objetivo é convidar um(a) engenheiro(a) para compartilhar sua trajetória, desafios, área de atuação, de modo a inspirar os(as) discentes. Este tipo de palestra já vem sendo 
realizada com sucesso na universidade pelo ramo estudantil e foi introduzida dentro da programação da Mentoria de Carreira. Durante o projeto, três engenheiras puderam compartilhar muito conhecimento aos participantes, como dicas de estudos, empreendedorismo e sua atuação no mercado de trabalho.

IV. Networking no IEEE: o IEEE se faz presente em muitas universidades brasileiras e tem como principal característica a sua rede de contatos, ou seja, o forte networking que os membros adquirem. Dessa forma foi apresentado neste webinar o que é o IEEE, como ser membro do instituto e a experiência em fazer parte desta grande rede, agregando muitos benefícios para a carreira acadêmica e profissional do(a) estudante universitário(a). A palestrante foi uma estudante de engenharia membra do IEEE e com grande experiência dentro das representações do instituto no Brasil.

V. Pesquisa Científica: buscando não focar apenas no desenvolvimento de carreira fora da universidade, foi abordada, também, a carreira acadêmica e como acontece a pesquisa científica nas universidades do Brasil. Foram abordados os passos para se tornar um(a) pesquisador(a), desde a graduação até o doutorado. A palestrante foi uma aluna de engenharia de alimentos com experiência em laboratório e pesquisas.

VI. Currículo e LinkedIn: neste workshop foi discutido sobre a composição do currículo, com dicas sobre como editá-lo, ferramentas que podem ser utilizadas e orientações de escrita. Além disso, foi mencionada a ferramenta LinkedIn (rede social profissional) para a construção de um perfil forte e redes de contatos. A palestrante foi uma estudante de engenharia de produção, com experiências com o tema discutido.

VII. Competições Universitárias: nesta live, foram convidadas três graduandas que atualmente participam ativamente de grupos de competições da UFPA, sendo estes: robótica competitiva, nautimodelismo e aerodesign. Foram discutidas as atividades que cada equipe realiza e desenvolve, como integrar os times e a importância de participar deste tipo de grupo.

VIII. Trainee na Prática: este workshop focou nos(as) universitários(as) que estão no final do curso ou já formados(as). Abordou todo o processo do Programa Trainee: inscrição no programa de diversas empresas no Brasil, as etapas dos processos seletivos, o que acontece depois de aprovado e a carreira do(a) trainee dentro das empresas. A engenheira eletricista convidada é trainee de uma grande empresa instalada no estado do Pará, aprovada em um processo seletivo nacional.

IX. Extensão e Empreendedorismo Social: buscando mostrar as atividades internas nas universidades, neste bate-papo foi discutida a importância de levar o conhecimento técnico adquirido nos cursos de graduação para a comunidade externa à universidade, ou seja, através dos Projetos de Extensão. Nesta live foi apresentada o time Enactus, um projeto extensionista e de empreendedorismo social, que tem representantes em várias instituições de ensino brasileiras e que leva às comunidades conhecimento e projetos de cunho empreendedor para empoderar economicamente as famílias que ali pertencem. Foi abordado o que essas atividades contribuem para os(as) alunos(as) de graduação e para os(as) alunos(as) já formados(as).

$\mathrm{X}$. Experiência Internacional: tratado em forma de webinar, esta temática abordou a experiência de uma estudante estrangeira em uma universidade brasileira, trazendo discussão sobre as diferenças culturais, as dificuldades, as realizações e o futuro após a formação na graduação.

XI. Organização Financeira: este workshop trouxe conhecimentos sobre investimentos, organização das finanças pessoais, hábitos para economizar e juntar dinheiro, mesmo sendo universitário(a). A engenheira de produção convidada atua na área de empreendedorismo e com experiência em investimento e finanças pessoais. 


\subsection{Tecnologias utilizadas}

Com o objetivo de utilizar plataformas online, gratuitas, simples e populares para a melhor alcance do projeto dentre o meio universitário, foram empregadas as seguintes tecnologias:

I. StreamYard: consiste em uma aplicação WEB para produção de lives, sem a necessidade de downloads adicionais, precisando apenas de um computador com microfone e câmera. (StreamYard, 2020).

II. YouTube: é uma plataforma que se baseia em quatro pilares: liberdade de expressão, direito à informação, direito à oportunidade e liberdade para pertencer, promovidos através de vídeos (Youtube, 2020). Esta ferramenta foi usada como transmissora das lives gravadas no StreamYard.

III. Facebook: empresa que cria tecnologias com o objetivo de conectar pessoas (Facebook, 2020). Dentre essas tecnologias estão as ferramentas Instagram e WhatsApp que foram utilizadas como canais para divulgação das palestras e palestrantes, horários e dias das lives e, também, como recurso de comunicação direta com os espectadores e inscritos na mentoria.

IV. Kahoot: a Kahoot tem como missão tornar o aprendizado lúdico. Esta plataforma facilita a criação, o compartilhamento e a reprodução de jogos de aprendizagem e questionários, em poucos minutos (Kahoot, 2020). Foi utilizada para a criação dos questionários relacionados ao conteúdo das lives. Para respondê-lo, os participantes apenas precisavam criar um usuário, sem a necessidade de instalar a aplicação em seu dispositivo eletrônico.

\section{RESULTADOS}

O principal objetivo do projeto Mentoria de Carreira Universitária foi promover conhecimentos essenciais e diferenciais para o início da carreira dentro da engenharia através da promoção de onze lives no período de um mês. Como resultado, o projeto alcançou 2400 visualizações, a partir de 765 espectadores, representando um aumento de $999 \%$ para o canal do IEEE UFPA na plataforma Youtube (Figura 1). Os dados gerados mostram que o alcance das lives foi muito satisfatório e com boa participação do público, que puderam interagir com cada palestrante durantes as lives.

Figura 1 - Alcance do Projeto Mentoria Universitária

\begin{tabular}{|c|c|c|c|}
\hline Impressões & Taxa de cliques de impressões & Visualizações & Espectadores únicos \\
\hline $16,7 \mathrm{mil}$ & $6,1 \%$ & 2,4 mil & 765 \\
\hline$\uparrow>999 \%$ & $\uparrow 43 \%$ & $\uparrow>999 \%$ & $\uparrow>999 \%$ \\
\hline
\end{tabular}

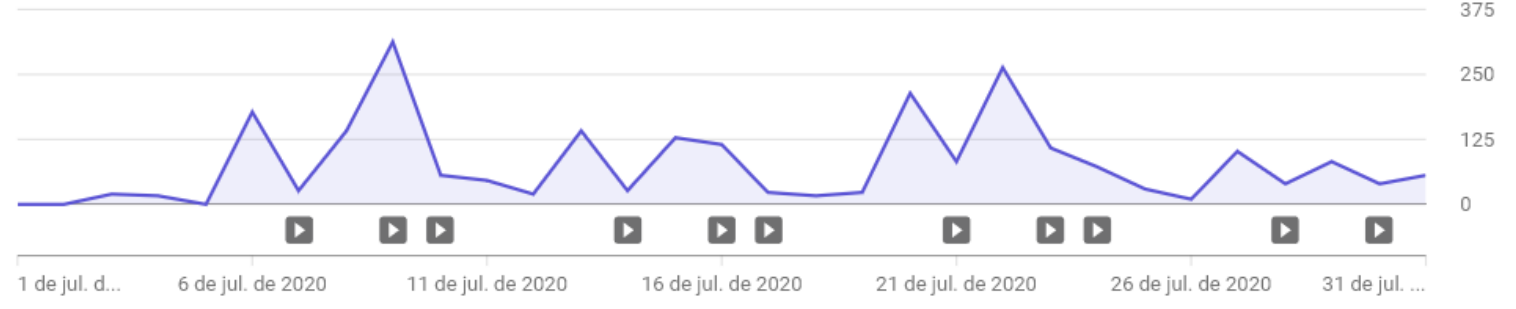

Fonte: Autor (2020) 
A estatística segundo idade e gênero, mostram maior participação feminina e uma faixa de idade majoritária entre 18 e 24 anos (Figura 2).

Figura 2 - Estatística do público segundo gênero e idade

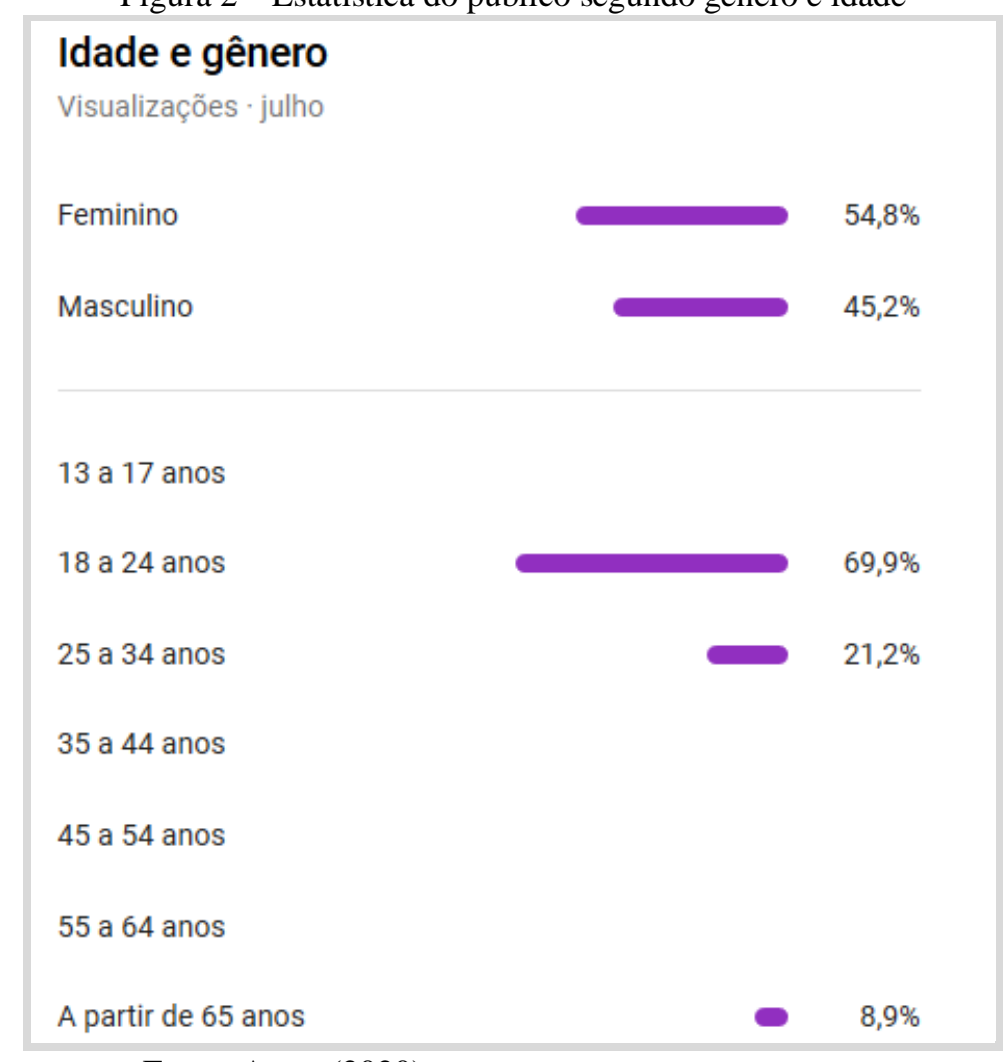

Fonte: Autor (2020)

\section{CONSIDERAÇÕES FINAIS}

A mentoria é uma prática diferencial que possibilita o contato de alunos com profissionais atuantes no mercado de trabalho, possibilitando a obtenção de conhecimentos essenciais para aqueles que estão no início de carreira. Visando este objetivo e se adequando ao novo contexto social devido à pandemia do novo coronavírus, o projeto Mentoria de Carreira Universitária possibilitou o contato de alunos(as) de todo o Brasil com engenheiras experientes e estudantes de engenharia, que são referência em atividades acadêmicas e extracurriculares, de forma online e gratuita. Além disso, o uso de elementos de jogos para avaliar e difundir os conhecimentos trabalhados durante a mentoria se mostrou uma estratégia eficaz para engajar os participantes durante o projeto, tornando as atividades mais atrativas.

Todas as lives promovidas estão disponibilizadas no canal do IEEE UFPA na plataforma Youtube.

\section{Agradecimentos}

Agradecimento ao Instituto de Engenheiros Eletricistas e Eletrônicos (IEEE), ao Ramo Estudantil IEEE UFPA, ao Grupo de Afinidade Mulheres na Engenharia (WIE UFPA), à Universidade Federal do Pará (UFPA) e à todas as engenheiras, empresas e alunas voluntárias do WIE que participaram deste projeto. 


\title{
REFERÊNCIAS
}

ARANTES, L.S.; VIEGAS, T. D. O. Mentoring para universitários: potencializando o desempenho profissional. Atlante Cuadernos de Educación y Desarollo. Outubro, 2018.

FACEBOOK. About Facebook. Disponível em: https://about.fb.com/. Acesso em: 7 ago. 2020.

IEEE. Mission and Vision. Disponível em: https://www.ieee.org/about/vision-mission.html. Acesso em: 1 ago. 2020.

KAHOOT. What is Kahoot!?./ Disponível em: https://kahoot.com/what-is-kahoot/. Acesso em: 7 ago. 2020.

RODRIGUES, Mellyna da Silva. Relações entre produtos audiovisuais e educação: Mídia e Ensino Durante a Pandemia de COVID-19. 2020. Trabalho de Conclusão de Curso Departamento de Comunicação Social, Universidade Federal do Rio Grande do Norte, Natal, 2020.

SILVA, R.; ALMEIDA, P. S. B. As Mudanças da Compreensão da Cognição a Partir do Uso das Novas Tecnologias da Informação e Comunicação na Aprendizagem. Revista Educação, Ciência e Inovação, v.4, n.1, p. 136-152, 2019.

TOLOMEI, B. V. A gamificação como estratégia de engajamento e motivação na educação. Revista Científica em Educação a Distância, v.7, n.2, p. 145-156, 2017.

STREAMYARD. About Us. Disponível em: https://streamyard.com/resources/about/. Acesso em: 7 ago. 2020.

YOUTUBE. YouTube About. Disponível em: https://www.youtube.com/intl/pt-BR/about/. Acesso em: 7 ago. 2020.

\section{THE ONLINE MENTORING PROJECT AS PROFESSIONAL CAREER GUIDANCE IN ENGINEERING}

\begin{abstract}
An important question that is treated in engineering education is the possibility of learning beyond the classroom and to develop not only technical skills. Thus, the affinity group IEEE UFPA Women in Engineering developed the Online Mentoring Project in order to contribute to the professional career development of IEEE members, volunteers, and the academic community. Through streams on the Youtube platform, this project promoted discussion about essential skills and competencies that a company seeks as well as internship programs and scientific projects that contribute to the career development of the engineering students. For one month, the project achieved 2400 views with eleven lives, divided into webinars, workshops, and technological coffee, all of these events promoted by female engineers and engineering students.
\end{abstract}

Keywords: IEEE. Professional Career. Mentoring. 